\title{
Entretien avec Christian Garcin
}

\section{OpenEdition}

\section{Journals}

Édition électronique

URL : http://journals.openedition.org/recherchestravaux/140

DOI : 10.4000/recherchestravaux.140

ISSN : 1969-6434

Éditeur

UGA Éditions/Université Grenoble Alpes

Édition imprimée

Date de publication : 15 avril 2006

Pagination : 135-140

ISBN : 2-9518254-8-X

ISSN : 0151-1874

Référence électronique

«Entretien avec Christian Garcin », Recherches \& Travaux [En ligne], 68 | 2006, mis en ligne le 06 novembre 2008, consulté le 08 septembre 2020. URL : http://journals.openedition.org/ recherchestravaux/140 ; DOI : https://doi.org/10.4000/recherchestravaux.140 


\section{Entretien avec Christian Garcin}

Les premières œuvres de Christian Garcin, dans les années 90, ont été des recueils de "vies brèves", Vidas en 1993 et L'Encre et la Couleur en 1997, dans la collection "L'Un et l'Autre» chez Gallimard, puis Vies volées chez Climats en 1999. Au centre de ces récits de vies, des personnages réels, d'autres imaginaires, et parmi eux de nombreux artistes: Van Eyck, le Caravage, des peintres italiens de la Renaissance, des peintres chinois.

Bien qu'il se soit éloigné depuis bientôt une dizaine d'années de l'écriture biographique pour explorer avec succès les territoires de la poésie, de la nouvelle et du roman, Christian Garcin a accepté de répondre par écrit à quelques questions posées par Brigitte Ferrato-Combe sur sa pratique originale de la fiction biographique et sur l'intérêt qu'il porte à la peinture et à l'histoire de l'art. Cet entretien a eu lieu à l'automne 2005.

Brigitte Ferrato-Combe - Vos premiers livres ont été des recueils de courts récits biographiques, de "vies brèves". C'est à cette époque également que vous avez écrit Piero ou l'équilibre, publié en 2004 aux éditions L'Escampette, ouvrage plus ample que vous définissez vous-même comme "essai biographique", et dont une version abrégée figure dans L'Encre et la Couleur sous le titre "Piero ou le consentement». Après l'avoir ainsi illustrée, vous semblez abandonner la fiction biographique ${ }^{1}$, au profit de la nouvelle, de la poésie et surtout du roman. Que représente ce genre pour vous?

Christian GARCIN - Le genre de la "vie brève" ou celui des "vidas" des troubadours - en somme ce que l'on appelle aujourd'hui "fiction biographique» - n'était pas pour moi un genre à proprement parler, plutôt une

1. Elle réapparaît cependant de manière ponctuelle et allusive dans les Deux fragments oubliés, publiés par Initiales à la fin de l'année 2005. 
constellation de textes qu'à une époque je fréquentais plus ou moins, en dilettante. Lorsque j'ai écrit Vidas, j'avais lu quelques vidas de troubadours, les Vies des douze Césars de Suétone, pas encore les Vies brèves d'Aubrey (ce serait pour plus tard, on en trouve l'écho dans le "Shakespeare» de Vies volées), et quelques extraits des Vies de Vasari. À quoi il faudrait ajouter, pour dresser un panorama complet, quelques-uns des Traités de Quignard qui flirtent parfois avec ce genre-là, ainsi que la Vie de Joseph Roulin, de Michon (mais pas les Vies minuscules). Mon projet fut d'écrire des vies de personnages illustres (César, le Caravage, Pythagore), ou qui l'ont été et ne le sont plus (Apollonios de Tyane), ou inconnus ayant existé (Jean Mouchon), ou purement imaginaires (Alain Gentil), et mêler le tout, gommer les différences entre eux en révélant les points communs, les hantises communes, la mort, etc. À la manière des troubadours - des vies plus ou moins imaginées -, et à la manière de Suétone - en mêlant le détail infime, l'anecdote, aux événements avérés (du moins pour ce qui concerne les figures illustres). Après avoir écrit Vidas, mais avant la correction d'épreuves, j'ai lu les Vies imagi naires de Schwob, et j'y ai trouvé quelques coïncidences étonnantes, comme le fait qu'il consacre une «vie» à Lesbia, l'amante de Catulle, qui figure aussi dans Vidas. J'ai donc décidé de rendre à Schwob un hommage a posteriori, j'ai rebaptisé les personnages de Vidas qui, purement imaginaires, n'avaient pas de nom (leur intitulé était «Anonyme»), et j'ai donné à l'un d'eux le nom d'un personnage de Schwob, Alain Gentil.

Après cette longue introduction je peux répondre à votre question, mais, je le crains, sans y répondre vraiment. Car au bout du compte je ne sais pas très bien ce que représente, ou représentait, ce "genre» pour moi. Sans doute, à cette époque-là, s'agissait-il de me situer, c'est-à-dire, d'une certaine manière, d'affirmer: c'est là que je suis, dans l'art, dans l'histoire, dans la littérature, c'est là qu'est mon véritable lieu. Écrire, c'était envoyer vers moi d'abord (au début je n'imaginais pas voir ces textes publiés) puis vers l'extérieur une image de moi qui me paraissait plus conforme à ce que j'étais que celle que je donnais à voir habituellement. Il s'agissait de me construire, et de délimiter un territoire. Pour cela, ces textes et cette forme, résolument non autobiographique (me semblait-il, car évidemment, rien n'est si simple), et ces modèles revendiqués. Par la suite, sans doute ai-je eu peur de la «recette». Je veux dire que j'ai eu le sentiment de pouvoir répéter à l'infini ce type de texte, selon ce modèle et dans ce ton, qui au début était si exactement le mien, et dont au fil du temps je me suis éloigné. De tout cela il me fallait sortir: je n'avais aucune envie de me spécialiser dans les «vies brèves». 
B. F.-C. - Dans les ouvrages des années 90, de nombreux textes témoignent d'un intérêt pour l'histoire de l'art, et même d'une familiarité certaine avec les perspec tives ouvertes par cette discipline, ou même avec ses modes de pensée. Quelle place l'histoire de l'art a-t-elle occupé, ou occupe-t-elle encore, dans votre parcours?

Ch. G. - Les rencontres déterminantes qui décident du choix d'une vie sont finalement assez rares. J'ai dédicacé Piero ou l'équilibre à quelqu'un [NDLR: Jacqueline Bretonnel] qui m'a, ainsi que le dit cette dédicace, "ouvert les yeux ", voici environ vingt-cinq ans. L'enseignement qui fut le sien a déterminé une bonne partie de ma vie par la suite, en déployant devant moi et de manière définitive le monde de l'art - et, plus généralement, celui de l'esprit humain, à la fois dans sa dimension historique et individuelle. C'était comme poser des jalons, de petites lanternes sur un chemin obscur et fascinant qui s'ouvrait devant moi. C'était surtout, je l'ai su tout de suite, multiplier ma vie. Ces maigres études achevées, je n’ai jamais cessé de fréquenter le monde de la peinture et de l'art, tant dans sa réalité contemporaine que dans sa dimension passée, historique ou littéraire.

\section{B. F.-C. - Certains historiens d'art vous ont-ils particulièrement marqué?}

Ch. G. - Ils sont nombreux. Les tout premiers furent Llonello Venturi (Pour comprendre la peinture) et René Huygue (L'Art et l'Âme, Sens et destin de l'art). Ensuite Élie Faure et Ernst Gombrich. Je cite d'abord ceux-là, parce qu'ils furent chronologiquement les premiers, mais aussi parce qu'ils proposaient une vision globalisatrice qui m'enchantait: Huygue (que je n'ai pas relu depuis) et Élie Faure (que je compulse toujours) surtout, dont la vue d'ensemble (regard d'aigle hégélien) sur le fourmillement des hommes et des pensées, la mise en relation des formes et des idées, l'audace de certains raccourcis, la force des intuitions, m'impressionnaient assez. Par la suite, j'ai lu Focillon, Francastel, Roberto Longhi ou André Chastel, et d'autres encore, mais sur des époques ou des sujets précis. Et, plus récemment, Georges DidiHuberman et Daniel Arasse.

B. F.-C. - Les Vies de Vasari sont très présentes dans vos textes. À quel moment, et dans quelle édition, les avez-vous lues? Vous arrive-t-il de les relire?

Ch. G. - J'ai découvert les Vies de Vasari en extraits, dans une vieille édition dénichée chez un bouquiniste. Je ne sais plus très bien quand je les ai lues. J'aurais dit spontanément: après avoir écrit Vidas, mais puisque la vie de Donatello en porte la trace, ce doit être avant, ou pendant, je ne sais plus très bien. Je ne les ai pas relues depuis. 
B. F.-C. - L'ouvrage de Vasari vous semble-t-il avoir joué un rôle dans le projet d'écrire des récits de vie? A-t-il pu être un modèle, un contre-modèle, un simple déclencheur de l'envie d'écrire sur ces peintres, une source d'anecdotes qui stimu lent le récit, à partir desquelles la fiction peut s'élaborer?

Avez-vous eu le projet de réécrire Vasari, ou d'écrire contre lui?

Ch. G. - Je sais que j'avais déjà écrit quelques textes sur des peintres (le Caravage, notamment), et je me disais que je devais lire ces Vies, ou certaines d'entre elles, car elles pourraient, pensais-je, alimenter d'autres textes. J'étais donc clairement à la recherche d'une «source d'anecdotes qui stimulent le récit».

"Masaccio» et "Piero" dans L'Encre et la Couleur viennent signifier cette dette en citant abondamment Vasari. Donatello dans Vidas également. Mais Vasari, s'il a été à la source d'anecdotes, n'a pas été à proprement parler un modèle (mes modèles, s'il doit y en avoir, étaient plutôt Suétone et les troubadours). Peut-être alors un contre-modèle? Par moments sans doute, mais surtout un matériau biographique parmi d'autres, disons, comme Aubrey pour Shakespeare, les «vidas" pour les troubadours, ou Suétone pour les empereurs.

B. F.-C. - Et la peinture chinoise... comment l'avez-vous découverte? Avez-vous lu des récits sur la vie de ces peintres?

Ch. G. - J'ai découvert la peinture chinoise à travers les livres de François Cheng, la pensée chinoise à travers Marcel Granet, François Jullien et, à nouveau, François Cheng. C'était un goût personnel, alimenté ensuite par des voyages, qui ont été sans doute déclencheurs de l'écriture. Les raisons pour lesquelles la Chine m'attirait sont multiples, j'ai tenté de les élucider dans un livre intitulé Itinéraire chinois, sans guère y parvenir. Disons qu'il doit y avoir une part de fantasme (ou de rêve), une part de biographique (ou de chromosomique), et sans doute une prédisposition générale de mon esprit à tout cela. L'Orient, chinois ou japonais, m'est de toute manière très proche; je dis parfois que je me sens chinois par mes racines paysannes, et japonais par mon imaginaire.

Le premier texte "chinois» que j'ai écrit, "Le coin et la moitié», est consécutif à la lecture de Vide et Plein de François Cheng, et surtout à mon premier voyage en Chine. Ensuite, $j$ 'ai lu d'autres livres sur ou autour de la peinture chinoise, mais aucun récit de vie de peintre. 
B. F.-C. - Dans L'Encre et la Couleur, peintres chinois et peintres italiens de la Renaissance se trouvent confrontés, dans l'alternance des récits qui leur sont consacrés. Des liens nombreux et imprévus se tissent à la lecture des uns aux autres. Qu'est-ce qui motive pour vous cette confrontation?

Ch. G. - Ce n'était pas un projet d'écriture, mais une conséquence de la nécessité d'organiser le livre. C'est tout simple, et à vrai dire sans grand intérêt: il se trouve que j'avais écrit des nouvelles sur des peintres chinois, d'autres sur des peintres italiens, mais sans jamais les relier les unes aux autres. Lorsque j'ai décidé d'assembler ces textes pour en faire un livre, il m’a semblé qu'une alternance Chine-Italie fonctionnerait bien. S'il y a des liens entre les textes chinois et italiens, je m'en réjouis, mais ce sont des jeux d'échos inconscients.

B. F.-C. - Voyez-vous une particularité au fait d'écrire sur la peinture? ou de raconter la vie d'un peintre, ou d'un sculpteur, plutôt que celle d'un écrivain comme vous l'avez fait aussi pour Shakespeare ou Agrippa d'Aubigné?

Ch. G. - Je ne sais pas si j'écris sur la peinture. Disons que j’ai écrit, parfois, sur des peintres. Le discours littéraire, ou littérarisant, sur la peinture, m'ennuie souvent. La vie des créateurs que j'admirais, ou dont je me sentais proche, m'a plus intéressé. Il y a des proximités. Il se trouve bien entendu qu'il peut m'arriver de me sentir plus proche de tel écrivain des siècles passés que de mes contemporains, ou d'un peintre que d'un écrivain. Le geste créateur en tout état de cause ne me semble pas très différent, que l'on soit peintre ou écrivain. Nous sommes plus ou moins confrontés aux mêmes problèmes: problèmes de fond, de forme, de structure; tenter d'organiser un certain désordre, restituer l'inconnu qui est en nous au moyen du connu. "L'art ne reproduit pas le visible, il rend visible», comme disait Klee. Ou bien, dit différemment, c'est " tenter de donner à la réalité les éclats confus de l'imaginaire» (Malraux). C'est cela qui m'importe.

B. F.-C. - Dans le roman Sortilège, Ezra, qui a fui la société des hommes et vit dans une grotte en plein désert, couvre des cahiers d'écriture et de dessins. Pratiquez-vous le dessin ou la peinture, ou la calligraphie?

Ch. G. - Non. C'est un de mes regrets. Enfant, je dessinais plutôt bien. Adolescent, je reproduisais les comics américains... J'avais même inventé de courtes BD (de tout cela je parle un peu dans J'ai grandi, qui paraît en jan- 
vier ${ }^{2}$ ). Mais si je savais très bien recopier, je ne savais pas dessiner vraiment. Je persiste à penser que le dessin ou la peinture sont un de mes possibles enfuis, et qu'il existe quelque part un autre espace-temps, parallèle à celui-ci, dans lequel les bifurcations de ma vie ont été autres, et où je suis peintre, ou dessinateur. Mais je ne le rejoindrai jamais. Et bien entendu, le Garcin de cet autre espace-temps tient le même discours au sujet de l'écriture.

2. J'ai grandi, Gallimard, «L'Un et l'Autre», janvier 2006. 